\title{
Three-dimensional Structure of Abrin-a A-chain Having Ribosome Inactivating Activity
}

\author{
By Tetsuya Kohno,*) Toshiya Senda, ${ }^{*}$ Hideki Narumi, ${ }^{* *)}$ Shigenobu Kimura,**) \\ and Yukio Mitsui*) \\ (Communicated by Setsuro EBASHI, M. J. A., March 13, 1995)
}

\begin{abstract}
Three-dimensional structure of abrin-a A-chain has been solved by X-ray crystal structure analysis. The protein is a member of the Type II ribosome inactivating proteins (RIPs) which catalyze endohydrolysis of the N-glycosidic bond of a particular adenosine at position 4,324 in $28 \mathrm{~S} \mathrm{rRNA}$ and thereby inhibit protein syntheses occurring on ribosome particles. It turned out that this enzyme had a similar three-dimensional structure to that of the A-chain of ricin, the crystal structure of which has been established before. The structure of abrin-a A-chain is composed of three domains, Domains 1, 2 and 3 . The active site is located in the cleft which is surrounded by the surfaces of Domains 1, 2 and 3 . The size of the cleft seems to be sufficient for recognizing RNA fragments including the GAGA tetraloop.
\end{abstract}

Key words : Abrin; crystal structure; ribosome inactivating protein; protein structure; ricin.

Abrin-a is a toxic protein isolated from the seeds of Abrus Precatorius and inactivates mammalian ribosomes. Besides abrin-a, many ribosome inactivating proteins (RIPs) from various plant seeds have been known. They are conventionally classified into two types: Type I and Type II. Type I RIPs are relatively abundant and nearly 30 kinds of them have been isolated so far. ${ }^{1)}$ By contrast, Type II RIPs are very rare, and until now sequences have been deposited in GenBank for only six of them. The target of the present study, abrin-a, belongs to Type II RIP.

Each of the Type II RIPs generally consists of two distinct polypeptide chains: the A-chain having an enzymatic activity and the B-chain which is a lectin providing the binding affinity to cell surfaces. Each of the Type I RIPs, on the other hand, consists of a single polypeptide chain. Abrin-a A-chain (ABRaA), as well as the A-chain of the other members of the Type II RIPs, catalyzes endohydrolysis of the Nglycosidic bond of a particular adenosine at position 4,324 in $28 \mathrm{~S}$ rRNA and thereby inhibits protein syntheses occurring on ribosome particles. ${ }^{2}$ This particular adenosine (A4324) is located on the highly conserved region of the $28 \mathrm{~S}$ rRNAs in many eukary-

*) Department of BioEngineering, Nagaoka University of Technology, Nagaoka, Niigata 940-21, Japan.

**) Basic Research Laboratories, Toray Industries, Inc., 1111 Tebiro, Kamakura, Kanagawa 248, Japan. otes. The region forms a double-helical stem with the sequence "GAGA" at the center of the region. ${ }^{2)-4)}$ A4324 is the second adenosine in the "GAGA" sequence. Type I RIPs show the same enzymatic activity as the A-chains of the Type II RIPs.

So far, three-dimensional crystal structures have been solved for several RIPs (both Type I and Type II). ${ }^{5)-13)}$ These studies have shown that the threedimensional structures of the A-chains of Type II RIPs are similar to the structures of Type I RIPs. However, intensive studies as to their substrate specificity and catalytic mechanism have been carried out only for the ricin system. ${ }^{3), 4), 14)-17)}$ Multiple alignment of amino acid sequences for the Type II RIPs reveals that the amino acid residues in the active site are almost fully conserved. On the other hand, the amino acid sequences in other regions show low homology among them. This fact suggests that the substrate specificity against the portions of the target RNA fragment involving the "GAGA" sequence may well be different among the Type II RIPs.

The potential utilization of the RIPs as reagents for treatment of cancer and AIDS has attracted much attention. ${ }^{11)}$ The RIPs can be targeted to specific cells by conjugating them to antibodies specific for the target cells. ${ }^{11)}$ To develop effective RIP reagents, detailed knowledge of the structure-activity relationship based on three-dimensional structure is neces- 
sary. To gain insights into the structure-activity relationship of $\mathrm{ABRaA}$, we undertook a crystallographic analysis of recombinant ABRaA.

We now report on the three-dimensional structure of the abrin-a A-chain (ABRaA) determined by X-ray crystal structure analysis. Crystallization of ABRaA was carried out using the hanging drop vapor diffusion method as described elsewhere (T. Kohno et al., submitted). The space group was $P 2_{1} 2_{1} 2_{1}$ with cell dimensions $a=74.8 \AA, b=83.2 \AA$ and $c=42.1 \AA$. Each asymmetric unit contains one molecule of ABRaA. The crystal structure was solved at $2.0 \AA$ resolution through a combination of molecular replacement (MR) and single isomorphous replacement (SIR) methods.

In the MR method, we used the crystal structure of ricin A-chain ${ }^{8)}$ as a search model. The solutions of the rotation and translation functions were easily found with the programs ALMN and TFFC in the CCP4 program suite. ${ }^{18)}$ Then, the structural model for ABRaA was constructed with the program QUANTA (Molecular Simulation Inc.). In this step, we made use of the preliminary coordinates of abrin-a A-chain as found in the whole abrin-a protein which is a heterodimer consisting of an A-chain and a B-chain. ${ }^{19)}$ The coordinates were kindly provided by Dr. Y.-C. Liaw. The polypeptide chain geometry as deduced from the coordinates provided by Dr. Liaw, however, were rather poor and the structure seemed to have some incorrect assignments of amino acid residues to the electron density map. We used the SA-omit map ${ }^{20)}$ to correct the models for ambiguous regions. Each of the omitted regions consisted of an $8 \AA$ sphere centered on each of the problematic residues. Due to poor electron density map, however, it was difficult to confirm whether the corrections made for the preliminary model were appropriate or not. At this stage, we decided to switch to a de novo phase determination based on a single isomorphous replacement (SIR) method. The uranyl acetate derivative was used for the SIR analysis. X-ray diffraction data for this derivative were collected by using an R-AXIS IIc area detector (Rigaku) with $\mathrm{CuK} \alpha$ radiation (generated by a Rigaku RU200 rotating-anode generator operating at $4.95 \mathrm{~kW}$ and focused by a SUPPER double focusing mirror). The difference Patterson map using a set of $|\mathrm{F}(+)-\mathrm{F}(-)|^{2}$ as the coefficients for Fourier transformation showed clear peaks at the same positions as those of usual isomorphous difference Patterson map indicating the accuracy of the measurement of anomalous dispersion. Accordingly we incorporated the anoma-

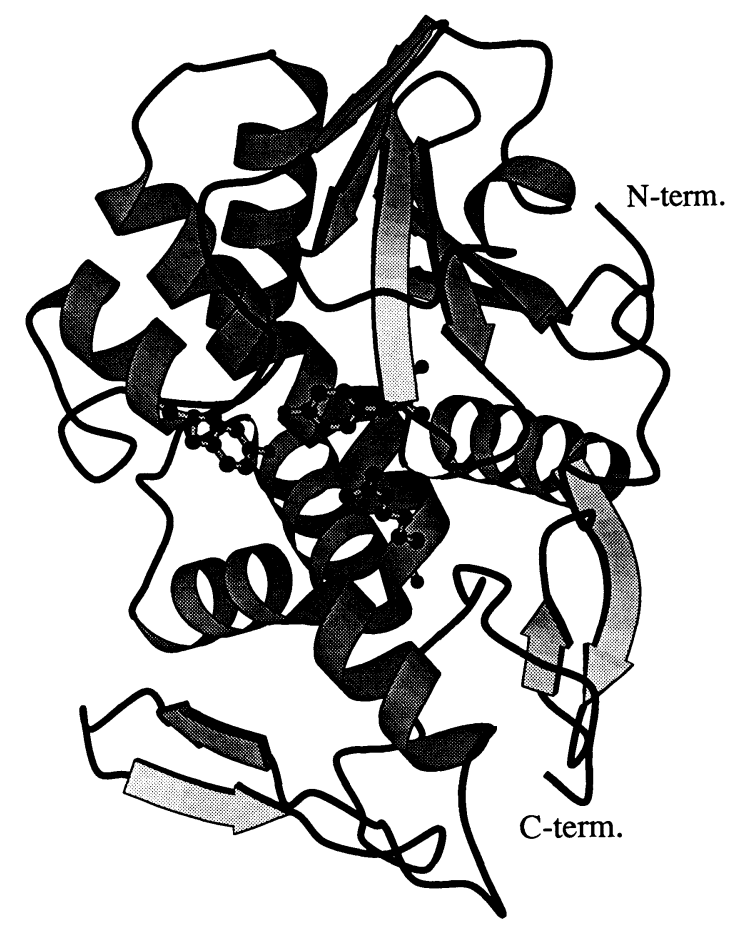

Fig. 1. The three-dimensional structure of abrin-a A-chain. Catalytic residues, Tyr74, Tyr 113 and Arg167, are shown as ball-and-stick model. $\mathrm{N}$ - and C-termini are labeled. $\alpha$ Helices and $\beta$-strands are shown as ribbons and arrows respectively. This figure was drawn with the program MOLSCRIPT. ${ }^{26)}$

lous dispersion effects to the phase calculation. The phases were calculated at $2.5 \AA$ resolution with program MLPHARE in the CCP4 program suite ${ }^{18)}$ resulting in the figure of merit of 0.44 . To further improve the electron density map, density modification procedures including solvent flattening, histogram matching and Sayer's equations were applied with the program SQUASH. ${ }^{21)}$ At the same time, the phases were extended up to $2.0 \AA$ resolution. In the above de novo phase determination, no phase information from the previous MR procedures was used. The resultant new electron density map was of sufficient quality to trace polypeptide chain folding. Based on this electron density map, we corrected the previous threedimensional structure model for ABRaA. It turned out that the coordinates provided by Dr. Y.-C. Liaw, although useful in the initial stage of the analysis, contained considerable amounts of error mainly centered on four misinterpreted regions.

The structure was refined by the program XPLOR. ${ }^{22), 23)}$ The current R-factor is $19.6 \%$ (free $\mathrm{R}$-factor is $27.0 \%$ ) for 14,518 reflections ( $74 \%$ of theoretical number) in the 8.0-2.0 $\AA$ resolution range. 


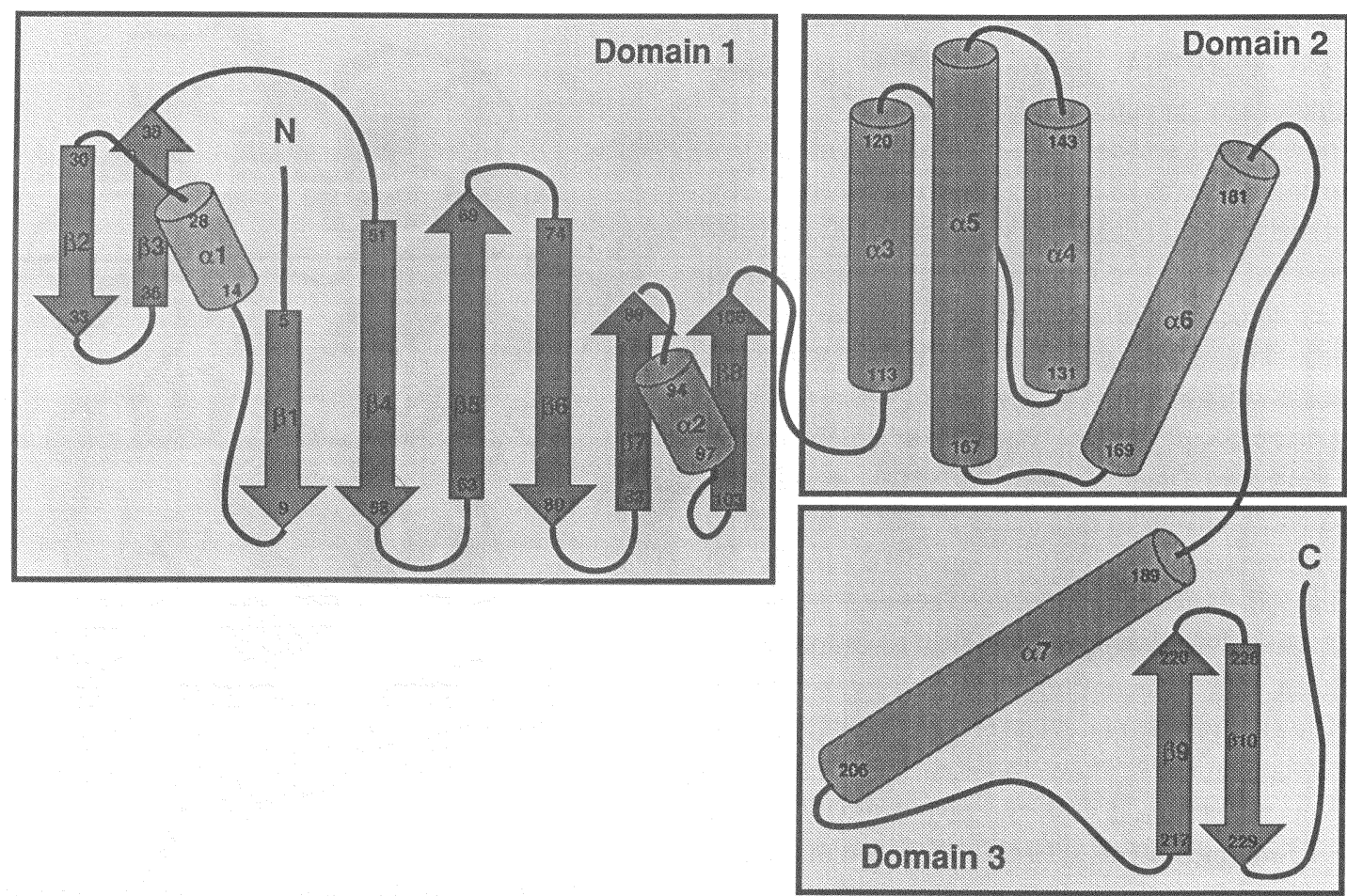

Fig. 2. Schematic representation of the abrin-a A-chain (ABRaA). $\beta$-Strands and $\alpha$-helices are represented by arrows and cylinders respectively. ABRaA is composed of three domains: Domains 1,2 and 3 .

The root-mean-square deviations from idealities were $0.010 \AA$ for bond lengths and $1.6^{\circ}$ for bond angles. The model involved 2,013 non-hydrogen protein atoms and 55 water molecules. Crystallographic details and the further refined structure will be published elsewhere. The refined coordinates of ABRaA will be deposited in the Brookhaven Protein Data Bank.

The overall structure of the ABRaA is shown in Fig. 1. The structure is very similar to that of ricin A-chain. ${ }^{8)}$ For comparison, the $\mathrm{C} \alpha$ chain of ABRaA and that of the A-chain of ricin were superimposed by noticing only the corresponding secondary structures for which regions the root-mean-square deviation of the $\mathrm{C} \alpha$ atoms was $1.46 \AA$. Compared with the amino acid sequence of ricin A-chain, there are two insertions and six deletions in that of the ABRaA chain. Examining the above superposition, it is clear that all the insertions and deletions occur at the loop region. As shown in Fig. 2, the three-dimensional structure of ABRaA is composed of three domains: Domain 1 (residues Glu1 to Gly111), Domain 2 (residues Thr112 to Asp188) and Domain 3 (residues Ala189 to Asn251). Domain 1 is a $\beta$-rich domain consisting of two $\beta$-sheets. The large $\beta$-sheet which is composed of $\beta 1, \beta 4, \beta 5, \beta 6$, $\beta 7$ and $\beta 8$ exhibits a left-handed twist when viewed perpendicular to the $\beta$-strand direction. Domain 2 is composed of four $\alpha$ helices, $\alpha 3, \alpha 4, \alpha 5$ and $\alpha 6$. There are many hydrophobic amino acid residues between Domains 1 and 2 . These hydrophobic interactions seem to be important to stabilize the three-dimensional structure of ABRaA. Finally Domain 3 is composed of a small $\beta$-sheet and a single $\alpha$-helix, $\alpha 7$. The $\alpha 7$ helix is kinked at the position of Asn197, the corresponding main chain dihedral angles being $\phi(197)=113.4^{\circ}$ and $\psi(197)=-122.1^{\circ}$. According to the preliminary structure of heterodimeric abrin-a as elucidated by Dr. Liaw, this part of the A-chain is in contact with the B-chain. In the superposition of the ABRaA chain and ricin A-chain as mentioned above, the $\beta$-sheet of Domain 3 does not fit well as the other part of the molecule. Most probably this fact occurs because the mode of interaction made by this $\beta$-sheet with the adjacent symmetry-related molecule is distinctly different between the two A-chains which lie in completely different crystal packing environments.

In view of the amino acid sequence homology and three-dimensional structural similarity, catalytic residues of ABRaA seem to be the same as those of ricin A-chain. The corresponding catalytic residues of ABRaA are Tyr74, Tyr113, Glu164 and Arg167. These 
residues have almost the same dispositions as those of ricin A-chain. This fact suggests that the same catalytic mechanism is adopted between ABRaA and ricin A-chain. The active site involving the abovementioned catalytic residues is located in the middle of the cleft of the ABRaA molecule. This cleft is surrounded by the surfaces of Domains 1,2 and 3. The dimensions of the cleft are $37 \AA$ (width) $\times 11 \AA$ (height) $\times 7 \AA$ (depth). The area appears to be large enough for recognizing GAGA tetraloop whose threedimensional structures have been established by NMR spectroscopy. ${ }^{22,23)}$ Furthermore the amino acid residues in the cleft are well conserved between ricin A-chain and ABRaA. This fact suggests that the cleft is the recognition site for the rRNA fragment involving the GAGA sequence. Hence the term "recognition cleft" would be appropriate to designate this area.

The amino acid residues on the molecular surface other than the "recognition cleft" are less conserved between the ricin A-chain and the ABRaA chain. Thus, if the sites of interaction between these A-chains and ribosomes as substrate were not confined to the mere GAGA tetraloop, the base sequence specificity in a broader sense may well be significantly different between the ricin A-chain and the ABRaA chain.

To investigate the enzymatic mechanism of ABRaA, a crystallographic analysis of ABRaA complexed with formycin-5'-monophosphate (FMP) as well as biochemical analyses of ABRaA is in progress.

Acknowledgement. We would like to thank Dr. Y.-C. Liaw at the Institute of Molecular Biology, Academia Sinica (Taipei) for providing us with the preliminary coordinates of the intact abrin-a A-chain prior to publication.

\section{References}

1) Stripe, F., Barbieri, L., Battelli, M. G., Soria, M., and Lappi, D. (1992) Bio/Technology. 10, 405-412.

2) Endo, Y., and Tsurugi, K. (1988) J. Biol. Chem. 263, 87358739.

3) Endo, Y., Glück, A., and Wool, I. G. (1991) J. Mol. Biol. 221, 193-207.

4) Glück, A., Endo, Y., and Wool, I. G. (1992) J. Mol. Biol. 226, 411-424.

5) Montfort, W., Villafranca, J. E., Monzingo, A. F., Ernst,
S. R., Katzin, B. J., Rutenber, E., Xuong, N. H., Hamlin, R., and Robertus, J. D. (1987) J. Biol. Chem. 262, 5398-5403.

6) Katzin, B. J., Collins, E. J., and Robertus, J. D. (1991) Proteins 10, 251-259.

7) Rutenber, E., Katzin, B. J., Collins, E. J., Mlsna, D., Ernst, S. R., Ready, M. P., and Robertus, J. D. (1991) Proteins 10, 240-250.

8) Mlsna, D., Monzingo, A. F., Katzin, B. J., Ernst, S. R., and Robertus, J. D. (1993) Protein Science 2, 429-435.

9) Monzingo, A. F., Collins, E. J., Ernst, S. R., Irvin, J. D., and Robertus, J. D. (1993) J. Mol. Biol. 233, 705-715.

10) Fraser, M. E., Chernaia, M. M., Kozlov, Y. V., and James, M. N. G. (1994) Nature Structural Biol. 1, 59-64.

11) Ren, J., Wang, Y., Dong, Y., and Stuart, D. J. (1994) Structure 2, 7-16.

12) Zhou, K., Fu, Z., Chen, M., Lin, Y., and Pan, K. (1994) Proteins 19, 4-13.

13) Husain, J., Tickle, I. J. and Wood, S. P. (1994) FEBS Lett. 342, 154-158.

14) Ready, M. P., Kim, Y., and Robertus, J. D. (1991) Proteins 10, 270-278.

15) Kim, Y., Mlsna, D., Monzingo, A. F., Ready, M. P., Frankel, A., and Robertus, J. D. (1992) Biochemistry 31, 3294-3296.

16) Kim, Y., and Robertus, J. D. (1992) Protein Eng. 5, 775779 .

17) Monzingo, A. F., and Robertus, J. D. (1992) J. Mol. Biol. 227, 1136-1145.

18) Collaborative Computational Project, Number 4. (1994) Acta Cryst. D50, 760-763.

19) Liaw, Y.-C., Tahirov, T., Chu, S.-C., Lu, T.-H., and Lin, Y. J. (1993) Acta Crystallogr. A49 (Supplement), PS03.05 .28 , p. 89.

20) Hodel, A., Kim, S.-H., and Brünger A. T. (1992) Acta Cryst. A48, 851-858.

21) Zhang, K. Y. J. (1993) Acta Cryst. D49, 213-222.

22) Brünger, A. T., Kuriyan, K., and Karplus, M. (1987) Science 235, 458-461.

23) Brünger, A. T. (1992b) X-PLOR, Version 3.1. A system for X-ray crystallography and NMR (New Haven, Connecticut: Yale University Press).

24) Orita, M., Nishikawa, F., Shimayama, T., Taira, K., Endo, Y., and Nishikawa, S. (1993) Nucleic. Acids Res. 21, $5670-5678$.

25) Szewczak, A. A., Moore, P. B., Chan, Y.-L., and Wool, I. G. (1993) Proc. Natl. Acad. Sci. U.S.A. 90, 9581-9585.

26) Kraulis, P. J. (1991) J. Appl. Crystallogr. 24, 946-950. 